\title{
The acute effects of walking exercise intensity on systemic cytokines and oxidative stress
}

\author{
Malcolm Brown ${ }^{1,2} \cdot$ Conor M. McClean ${ }^{1} \cdot$ Gareth W. Davison ${ }^{1} \cdot$ John C. W. Brown ${ }^{1} \cdot$ Marie H. Murphy ${ }^{1}$
}

Received: 17 January 2018 / Accepted: 1 July 2018 / Published online: 14 July 2018

(c) The Author(s) 2018

\begin{abstract}
Purpose Oxidative stress is associated with tissue cytokine secretion although the precise mechanism(s) underpinning this relationship during high intensity intermittent exercise remains unclear. This study investigates the acute response to a bout of high intensity intermittent walking (HIIW), compared to continuous moderate intensity walking (CMW), on various cytokines and biomarkers of oxidative stress.

Methods Seventeen $(n=17)$ apparently healthy male participants (aged $22.6 \pm 4.6$ years; $\dot{V} \mathrm{O}_{2}$ max: $53.7 \pm 7.1 \mathrm{ml} \mathrm{kg}^{-1} \mathrm{~min}^{-1}$ ) undertook a randomised crossover study consisting of two exercise trials: (1) HIIW requiring $3 \times 5$ min bursts at $80 \%$ $\dot{V} \mathrm{O}_{2} \max$ (each separated by 5 min of walking at $30 \% \dot{V} \mathrm{O}_{2} \max$ ) and (2) $\mathrm{CMW}\left(60 \% \dot{\mathrm{V}} \mathrm{O}_{2} \max\right.$ for $30 \mathrm{~min}$ ). Each trial was separated by 7 days. Venous blood samples were obtained pre-exercise, post-exercise and at 2, 4, 24 and $48 \mathrm{~h}$ post-exercise for determination of systemic inflammation (IL-6 and TNF- $\alpha$ ), lipid soluble antioxidants and oxidative stress $\left(\mathrm{LOOH}, \mathrm{H}_{2} \mathrm{O}_{2}\right.$ and the ascorbyl free radical).

Results Both IL- 6 and TNF- $\alpha$ increased immediately post exercise, regardless of intensity and remained elevated until at least $4 \mathrm{~h}$ (main effect for time; $p<0.05$ ). While there was no change in either lipid peroxidation or free radical metabolism (Asc- and $\mathrm{H}_{2} \mathrm{O}_{2}$ ), $\alpha$-tocopherol increased (pooled HIIW and CMW, $p<0.05$ ), whereas lycopene decreased at $2 \mathrm{~h}$ post HIIW $(p<0.05)$.

Conclusion Bouts of both HIIW and CMW promote cytokine secretion post exercise, and this seems to be independent of oxidative stress. Further investigation is required to assess how such changes may underpin some of the transient health benefits of exercise.
\end{abstract}

Keywords Walking $\cdot$ High intensity intermittent exercise $\cdot$ Oxidative stress $\cdot$ Cytokine

$\begin{array}{ll}\text { Abbreviations } \\ \text { ANOVA } & \text { Analysis of variance } \\ \text { Asc. } & \text { Ascorbyl radical } \\ \text { CMW } & \text { Continuous moderate walking } \\ \text { DMSO } & \text { Dimethyl sulfoxide } \\ \text { DNA } & \text { Deoxyribonucleic acid } \\ \text { EDTA } & \text { Ethylenediaminetetraacetic acid }\end{array}$

Communicated by Fabio Fischetti.

Malcolm Brown

m.brown@qub.ac.uk

1 Sport and Exercise Sciences Research Institute, Ulster University, Jordanstown, County Antrim BT37 0QB, Northern Ireland, UK

2 School of Nursing and Midwifery, Medical Biology Centre, Queen's University Belfast, Belfast BT9 7BL, Northern Ireland, UK
ELISA Enzyme-linked immunosorbent assay

EPR Electron paramagnetic resonance

$\mathrm{H}_{2} \mathrm{O}_{2} \quad$ Hydrogen peroxide

HIIW High intensity intermittent walking

HPLC High performance liquid chromatography

IL- Interleukin

LOOH Lipid hydroperoxides

MAPK Mitogen-activated protein kinase

NF-кB Nuclear factor kappa B

$\mathrm{P} \quad$ Statistical significance

ROS Reactive oxygen species

RPM Revolutions per minute

SEM Standard error of the mean

TNF- $\alpha$ Tumour necrosis factor alpha

$\dot{V} \mathrm{O}_{2}$ max Maximal oxygen uptake 


\section{Introduction}

Recent research postulates that balanced redox status and chronic systemic low-grade inflammation are potent mediators of homeostasis (Gleeson et al. 2011). Exercise has the capacity to transiently provoke a response in both biological systems and as such, has received increased research attention (Steinberg et al. 2007). It is now well established that enhanced cellular respiration during exercise leads to the generation of partially reduced oxygen derivatives, termed reactive oxygen species (ROS) (Davison et al. 2012). ROS react indiscriminately causing damage to molecular components and may serve as key proponents in several diseases (Sies 2015). Normally antioxidant mechanisms effectively reduce their potency although if abundantly created, coping capacities become overwhelmed and a state of oxidative stress ensues (Powers and Jackson 2008).

While excessive amounts of ROS are detrimental, transient exercise-induced changes are now recognised as integral agents in promoting adaptation (Radak et al. 2013). Cellular stress (i.e. disruption to cellular homeostasis and not just oxidative stress) may serve as a key stimulant of 'myokine' expression and the downstream anti-inflammatory cascade (Scheele et al. 2009). Direct empirical evidence of this supposed action is limited, and somewhat controversial, yet several reviews support this plausible mechanism (Scheele et al. 2009; Welc and Clanton 2013; Peake et al. 2015; Ost et al. 2016). The underlying molecular processes remain equally unexplored, but may arise from oxidative or endoplasmic reticulum stress, subsequent induction of p38 mitogen-activated protein kinase (MAPK) and putative transcriptional regulation of NF- $\kappa \mathrm{B}$ (nuclear factor kappa B) (Scheele et al. 2009; Powers et al. 2011; Welc and Clanton 2013, Ost et al. 2016). Several reports advocate that $\mathrm{p} 38 \mathrm{MAPK}$ is activated in response to the physiological perturbations associated with acute high-intensity exercise, and the corresponding increases in muscular ROS (Kefaloyianni et al. 2006; Kramer and Goodyear 2007). Likewise, NF-кB is phosphorylated by ROS, promoting nuclear translocation and transcription of various cytokines and chemokines (Powers et al. 2011; Chu 2013; He et al. 2016). Thus muscular cytokine kinetics appear redox sensitive and given both share overlapping signalling pathways, conceivably ROS directly influence a parallel rise in cytokine secretion (Sallam and Laher 2016). Although at this time, little is known of the impact of exercise intensity and pattern on the interactive oxidative and inflammatory mechanisms, especially in the post-exercise period.

A rise in IL-6 secretion has been consistently reported in venous circulation following exercise, proportionally related to exercise intensity, duration and muscle mass recruited (Petersen and Pedersen 2005). Normally prolonged or unaccustomed bouts of exercise report the greatest increase, although a modest rise has been detected with moderate intensity walking (Nieman et al. 2005; Mendham et al. 2011). IL-6 may mediate the immunosuppressive cascade and influence metabolic activity, however the transient effects of a single bout of HIIW remain unclear, even though several cardioprotective effects have been identified using similar strategies (Hood et al. 2011; Little et al. 2011a).

Intense, interval based exercise has recently emerged as a time efficient strategy to elicit a range of health and fitness benefits including improved metabolism and immunity, oxidative capacity and antioxidant defence (Little et al. 2011b; Gibala et al. 2012). Previously published studies examining the impact of intense, interval exercise often deploy Wingate-like testing procedures using cycle ergometers, which may be poorly tolerated (Boutcher 2011). There is now a need for more practical strategies and in recent years effective models (i.e. decreasing absolute intensity, increasing duration and reducing resting intervals) have been presented (Leggate et al. 2010; Gibala et al. 2012). In fact, walking satisfies all three criterion and exercise intensity can be readily manipulated by increasing and decreasing cadence (Murphy et al. 2007). The threshold range for high intensity intermittent exercise has since been reduced, previously from near maximal bursts, to bouts beyond $75 \% \dot{\mathrm{VO}} \mathrm{O}_{2}$ max now deemed sufficient to induce a host of health benefits (Francois and Little 2015). Currently, there is a paucity of evidence characterising the transient response of cytokines and oxidative stress following differing walking intensities, particularly the influence of HIIW on these key biochemical parameters. Thus the aim of this study was to measure the differing biochemical response to single bouts of intermittent and continuous walking.

\section{Methodology}

\section{Participant characteristics}

Following ethical approval from the University Ethics committee and in accordance with the Declaration of Helsinki, seventeen $(n=17)$ apparently healthy and recreationally active (approximately 2 -h week ${ }^{-1}$ exercise) male participants $\left(22.6 \pm 4.6\right.$ years; $179.2 \pm 5.6 \mathrm{~cm} ; 79 \pm 10.6 \mathrm{~kg} ; \dot{V} \mathrm{O}_{2} \max :$ $53.7 \pm 7.1 \mathrm{ml} \mathrm{kg}^{-1} \mathrm{~min}^{-1}$ ) were recruited. Prior to commencing the study, all participants completed a health history questionnaire to ensure suitability and provided informed consent after full study details were disclosed. All participants were non-smokers and free from medication and antioxidant supplementation. 


\section{Experimental design}

Participants completed a randomised (number generated) crossover study consisting of two trials: (1) intermittent vigorous walking exercise (HIIW) and (2) continuous moderate walking exercise (CMW). Each trial was separated by 7 days and participants were asked to refrain from exercise and alcohol consumption $24 \mathrm{~h}$ prior to each trial. Participants recorded their dietary intake $24 \mathrm{~h}$ prior to testing and were asked to replicate this for the second trial. Participants were tested following a standard $10 \mathrm{~h}$ overnight fast.

\section{Maximal oxygen consumption}

Prior to experimental trials, participants undertook an incremental $\dot{V} \mathrm{O}_{2}$ max test on a motorised treadmill (H-P Cosmos, Germany) to exhaustion. Oxygen uptake was measured using a standard calibrated laboratory gas analysis system (Cosmed Quarkb ${ }^{2}$, Italy). Heart rate (Polar Electro, Finland) and perceived exertion was monitored continuously with a valid $\dot{V} \mathrm{O}_{2}$ max confirmed using the following criteria (1) the respiratory exchange ratio $\geq 1.15$ (2) a clear plateau in mean oxygen uptake $\left(<2 \mathrm{ml} \mathrm{kg}^{-1} \mathrm{~min}^{-1}\right)$ and (3) a heart rate within 10 beats $\mathrm{min}^{-1}$ of age predicted maximum (208-0.7 × age) (Howley et al. 1995).

\section{Exercise protocol}

Participants completed bouts of HIIW $(3 \times 5 \mathrm{~min}$ at $80 \%$ $\dot{V} \mathrm{O}_{2}$ max separated by $3 \times 5$ min at $30 \% \dot{V} \mathrm{O}_{2}$ max) and CMW $\left(60 \% \dot{V} \mathrm{O}_{2} \max\right.$ for $\left.30 \mathrm{~min}\right)$ in a random order. The vigorous bout was devised in accordance with recent recommendations (Gibala et al. 2012; Francois and Little 2015) and finalised following a pilot trial. Participants were instructed to walk for the entire exercise duration. The speed and incline of the treadmill was adjusted to ensure each participant achieved and remained at the desired intensity.

\section{Biochemical analysis}

\section{Venous blood sampling}

Venous blood samples were obtained at baseline, immediately post exercise, 2 and $4 \mathrm{~h}$ post exercise via a 22-gauge intravenous cannula (Biovalve Safe, Vygon, UK) inserted into a prominent antecubital fossa vein. Blood was drawn into serum clot activator and $\mathrm{K}_{3}$ EDTA vacutainers (Greiner Bio-One, Austria). Following collection, serum tubes were allowed to clot at room temperature for approximately 10 min while $\mathrm{K}_{3}$ EDTA tubes were placed on ice. Blood tubes were then centrifuged at $3500 \mathrm{rpm}$ for $10 \mathrm{~min}$ at $4{ }^{\circ} \mathrm{C}$ (Hettich, Germany). Serum and plasma were extracted and stored at $-80{ }^{\circ} \mathrm{C}$ prior to biochemical analysis. Follow up sampling also occurred at 24 and $48 \mathrm{~h}$ post exercise using venepuncture.

\section{Measurement of cytokines (IL-6 \& TNF-a)}

IL- 6 and TNF- $\alpha$ were determined in plasma by enzymelinked immunosorbent assay (ELISA) according to the manufacturer instructions (BioLegend, CA, USA). Absorbance was read spectrophotometrically using a microplate reader (EL 808, BioTek Instruments, USA) at $450 \mathrm{~nm}$ in conjunction with Gen5 software. Concentration was calculated using curve-fitting software with a 4-parameter logistics algorithm (ReadFit Pro 2014, Hitachi Solutions).

\section{Measurement of endothelin-1}

Endothelin-1 was determined pre and immediately post exercise in serum using a Quantikine ${ }^{\circledR}$ ELISA (R \& D Systems, UK). The absorbance was measured at $450 \mathrm{~nm}$ using a microplate reader (EL808, BioTek Instruments, USA). Concentration was calculated using curve-fitting software with a 4-parameter logistics algorithm (ReadFit Pro 2014, Hitachi Solutions).

\section{Measurement of lipid hydroperoxides (LOOH)}

Lipid hydroperoxides were measured in serum using the ferrous iron/xylenol orange (FOX) assay. The reagent was prepared using $25 \mathrm{mM} \mathrm{L}^{-1}$ of sulphuric acid $\left(\mathrm{H}_{2} \mathrm{SO}_{4}\right)$, $250 \mu \mathrm{M} \mathrm{L}^{-1}$ of ammonium ferrous sulphate, $100 \mu \mathrm{M} \mathrm{L}^{-1}$ of sorbitol and $100 \mu \mathrm{M} \mathrm{L}^{-1}$ of xylenol orange in distilled water. $90 \mu \mathrm{L}$ of serum was mixed with $900 \mu \mathrm{L}$ of reagent, incubated at room temperature for $30 \mathrm{~min}$ in darkness and read at $560 \mathrm{~nm}$ using a spectrophotometer (UV mini-1240 Shimadzu, Mason Technologies, Ireland).

\section{Measurement of hydrogen peroxide $\left(\mathrm{H}_{2} \mathrm{O}_{2}\right)$}

$\mathrm{H}_{2} \mathrm{O}_{2}$ was analysed in serum using an OxiSelect ${ }^{\mathrm{TM}}$ colorimetric assay according to the manufacturer instructions (Cell Biolabs Inc, CA, USA). The absorbance was immediately read at $560 \mathrm{~nm}$ by a microplate reader (FLUOstar Omega, BMG LABTEC, Germany).

\section{Electron paramagnetic resonance (EPR) spectroscopic analysis}

The ascorbyl radical was measured in plasma using EPR spectroscopy. $1 \mathrm{~mL}$ of plasma was mixed thoroughly with $1 \mathrm{~mL}$ of dimethyl sulfoxide (DMSO) in a glass test tube. $1 \mathrm{~mL}$ of solution was then drawn into a sterile syringe and flushed into the EPR cavity. All samples were analysed at room temperature using a calibrated Bruker EMX series 
X-band EPR spectrometer (Bruker, Germany). The spectrometer parameter conditions were set as follows: frequency $(9.785 \mathrm{GHz})$; microwave power $(20 \mathrm{~mW})$; modulation frequency $(100 \mathrm{kHz})$ and modulation amplitude $(1.194 \mathrm{G})$ for three sweeps. Spectral parameters were obtained using commercially available software (Bruker Win EPR System, Version 3.2) and filtered identically. The relative concentration of ascorbyl radical was determined by measuring signal intensity.

\section{Determination of lipid soluble antioxidants}

Endogenous lipid soluble antioxidants were measured using the simultaneous high performance liquid chromatography (HPLC) assay of Thurnham et al. (1988). Plasma samples were measured under the same testing parameters outlined in McClean et al. (2015) for $\alpha$-tocopherol, $\gamma$-tocopherol, retinol, lycopene, $\alpha$-carotene and $\beta$-carotene at changing wavelengths of 292, 325 and $450 \mathrm{~nm}$. Results were interpreted by Empower software (Version 2, Waters Corp, USA).

\section{Statistical analysis}

Statistical analysis was performed using SPSS version 22 (IBM, Hampshire, UK). Data were analysed using a twoway repeated measures analysis of variance (ANOVA) with one between (trial) and one within (time) subject factor. For significant interaction effects, within subject factors were further analysed using a Bonferroni-corrected paired samples $t$ test $(p<0.025)$. Between subject differences were analysed by one-way ANOVA with a posteriori Tukey Honestly Significant Difference (HSD) test. In the event of a main effect for time, data was pooled and paired sample $t$ tests were performed. The alpha level was set at $p<0.05$. A prospective power calculation, factoring critical difference, was conducted using the Altman method (Altman 1980). Retrospective power calculations were carried out while using SPSS. All data within are expressed as mean \pm SEM (standard error of the mean) unless otherwise stated.

\section{Results}

\section{Cytokine response}

Increases in IL-6 were observed following exercise in both conditions but no significant differences were observed between the trails (main effect for time, $p<0.05$; time $\mathrm{x}$ group interaction, $p>0.05$ ). Compared to pre-exercise, IL-6 increased at post exercise, 2 and $4 \mathrm{~h}(p<0.05)$ before returning to baseline by $24 \mathrm{~h}$ (Fig. 1). Peak IL-6 occurred immediately post exercise. Likewise, no changes were observed between groups for TNF- $\alpha$ over time $(p>0.05)$. There was, however, a main effect for time $(p<0.05)$ as TNF- $\alpha$ increased from baseline throughout the post-exercise period $(p<0.05)$ (Fig. 2). TNF- $\alpha$ peaked immediately post exercise (mean: $3.36 \mathrm{pg} / \mathrm{ml}$ ) with similar concentrations again at $4 \mathrm{~h}$ (mean: $3.34 \mathrm{pg} / \mathrm{ml}$ ).

\section{Endothelin-1}

There was no significant change from pre to post exercise between conditions (time $\times$ group interaction, $p>0.05$ ). A main effect for time $(p<0.05)$ was detected as endothelin-1 increased post exercise in both trials $(p<0.05)$ (Fig. 3).
Fig. 1 Effects of walking intensity on IL-6 over time $(n=17)$. Main effect for time at post exercise, $2 \mathrm{~h}$ and $4 \mathrm{~h}$ versus baseline $(p<0.05$; pooled HIIW and CMW data)

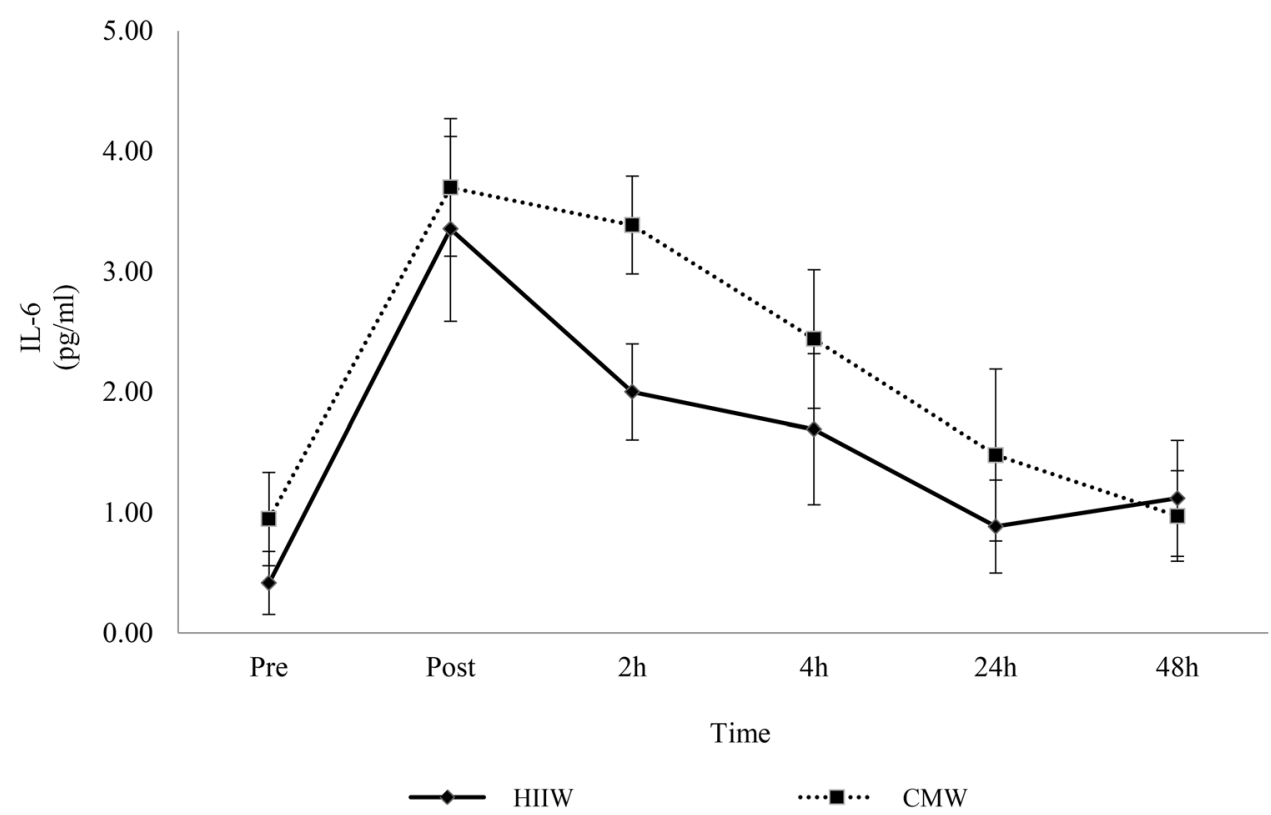


Fig. 2 Effects of walking intensity on TNF- $\alpha$ over time ( $n$ 17). Main effect for time at post exercise, $2,4,24$ and $48 \mathrm{~h}$ versus baseline $(p<0.05$; pooled HIIW and CMW data)

Fig. 3 Effects of exercise intensity on endothelin-1 over time ( $n$ 17). Main effect for time at post exercise versus baseline $(p<0.05$; pooled HIIW and CMW data)
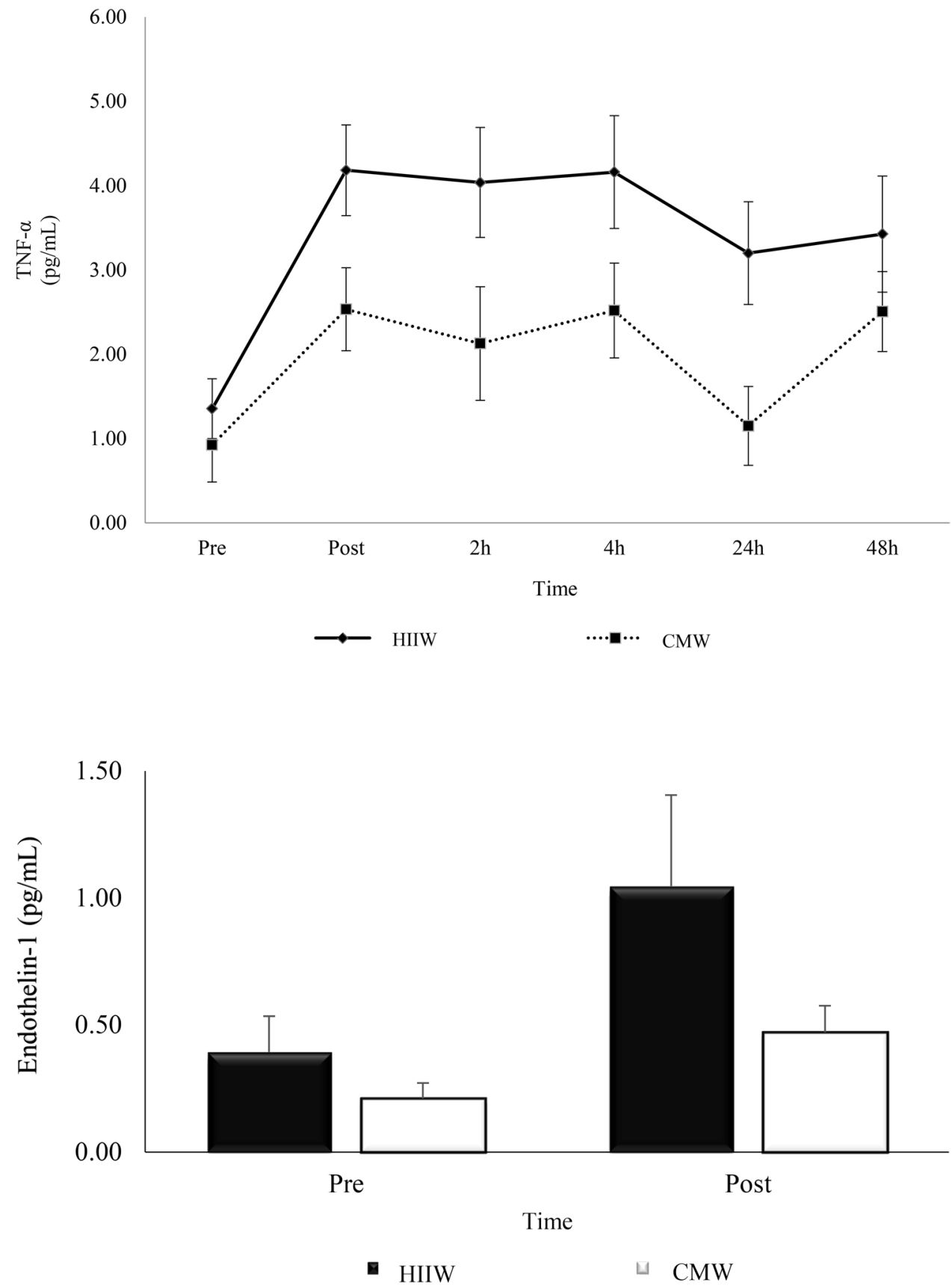

Table 1 Biomarkers of oxidative stress following exercise over time $(n=17)$

\begin{tabular}{llllllcc}
\hline Biomarker & Trial & Pre & Post & $2 \mathrm{~h}$ & $4 \mathrm{~h}$ & $24 \mathrm{~h}$ \\
\hline LOOH $\left(\mu \mathrm{M} \mathrm{L}^{-1}\right)$ & HIIW & $1.14 \pm 0.05$ & $1.19 \pm 0.05$ & $1.23 \pm 0.05$ & $1.22 \pm 0.05$ & $1.20 \pm 0.05$ & $1.19 \pm 0.04$ \\
& CMW & $1.22 \pm 0.07$ & $1.27 \pm 0.05$ & $1.23 \pm 0.04$ & $1.25 \pm 0.06$ & $1.20 \pm 0.05$ & $1.17 \pm 0.06$ \\
$\mathrm{H}_{2} \mathrm{O}_{2}(\mu \mathrm{M} / \mathrm{L})$ & HIIW & $39.4 \pm 3.61$ & $41.4 \pm 3.72$ & $41.7 \pm 3.64$ & $44.9 \pm 3.34$ & $38.0 \pm 3.95$ & $39.5 \pm 3.58$ \\
& CMW & $39.4 \pm 3.86$ & $42.1 \pm 3.56$ & $43.6 \pm 3.53$ & $42.8 \pm 3.33$ & $38.9 \pm 3.52$ & $41.1 \pm 2.98$ \\
Asc. (arbitrary units) & HIIW & $340 \pm 65$ & $366 \pm 61$ & $426 \pm 68$ & $381 \pm 77$ & $428 \pm 85$ & $352 \pm 78$ \\
& CMW & $447 \pm 80$ & $447 \pm 84$ & $557 \pm 78$ & $473 \pm 77$ & $544 \pm 70$ & $439 \pm 75$ \\
\hline
\end{tabular}

$C M W$ continuous moderate, $H I I W$ high intensity intermittent walking 


\section{Biomarkers of oxidative stress/damage}

Table 1 shows no changes in any markers of oxidative stress (LOOH, $\mathrm{H}_{2} \mathrm{O}_{2}$ and ascorbyl radical) within or between conditions over time $(p>0.05)$.

\section{Lipid soluble antioxidants}

There was no change for selected lipid soluble antioxidants (retinol, $\gamma$-tocopherol, $\alpha$-carotene and $\beta$-carotene) over time $(p>0.05)$. A main effect for time was detected for $\alpha$-tocopherol and further analysis suggests an increase at 2 and $4 \mathrm{~h}$ versus baseline ( $p<0.05$, Fig. 4 ). In addition, analysis of lycopene showed a time $\times$ group interaction effect $(p<0.05)$. Lycopene decreased $2 \mathrm{~h}$ post exercise during the high intensity intermittent walking trial compared to baseline and post-exercise $(p<0.05)$ (Fig. 5).

\section{Discussion}

This study is the first to examine the transient effects of HIIW on biomarkers of oxidative stress and inflammation over time. While no significant changes were observed, IL-6, TNF- $\alpha$ and endothelin-1, all increased post exercise irrespective of exercise intensity or pattern. Such changes were accompanied by an antioxidant response, whereby $\alpha$-tocopherol rose (HIIW and CMW data) and lycopene decreased (HIIW; pre- and post-exercise versus $2 \mathrm{~h}$ ).
Fig. 4 Effects of walking intensity on $\alpha$-tocopherol over time ( $n$ 17). Main effect for time at 2 and $4 \mathrm{~h}$ versus baseline $(p<0.05$; pooled HIIW and CMW data)

Fig. 5 Effects of walking exercise intensity on lycopene over time ( $n$ 17). Lycopene decreased at $2 \mathrm{~h}$ versus baseline $(*)$ and post-exercise (\#) $(p<0.05)$ in the HIIW trial only
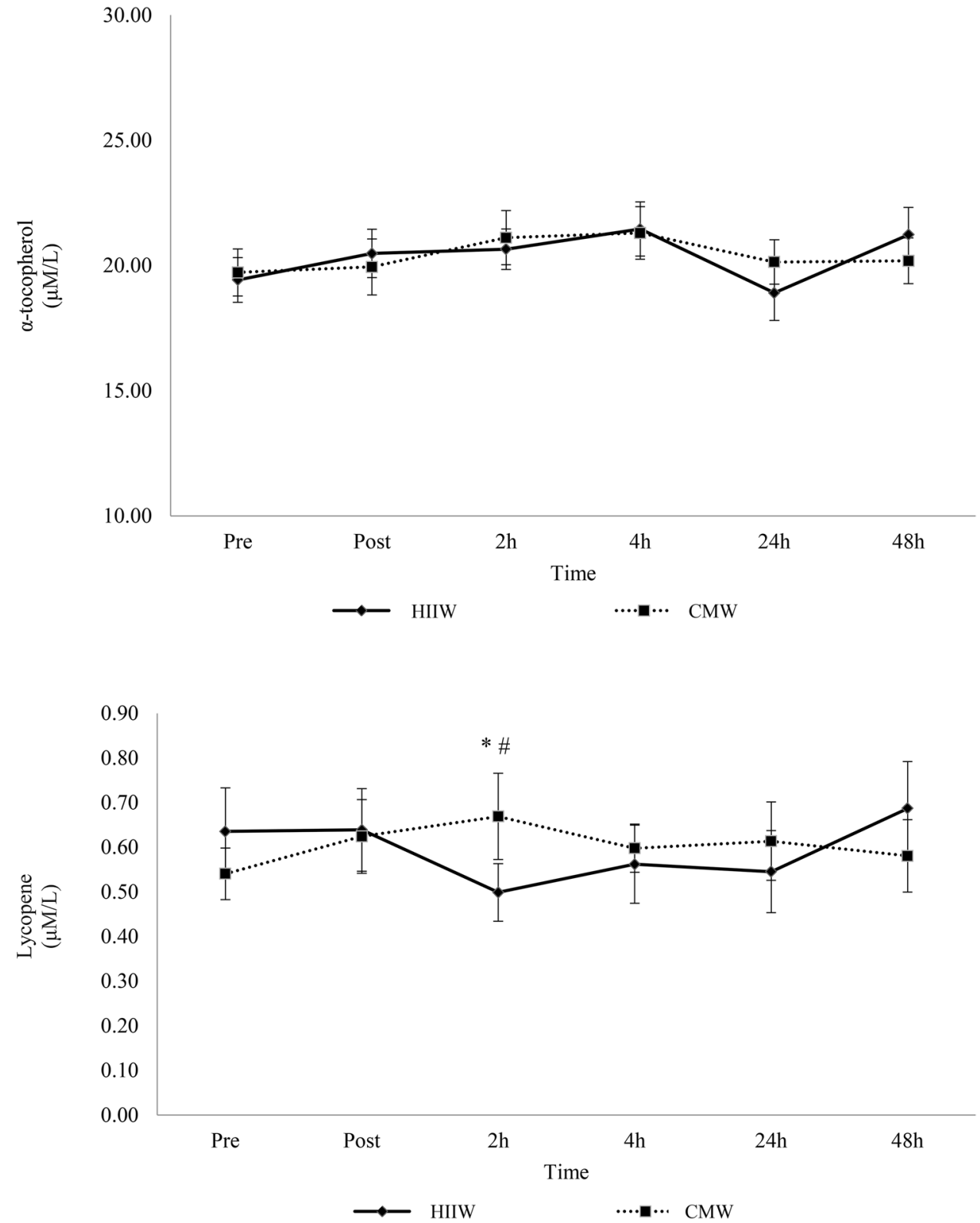
A main finding of this study is a bout of walking, regardless of intensity or pattern, promoted a systemic increase in IL- 6 and TNF- $\alpha$. Both cytokines peaked immediately post exercise, remained elevated at $4 \mathrm{~h}$ and decreased thereafter. These results complement other findings that have shown a rise in plasma IL-6 following both moderate and vigorous exercise (Harris et al. 2008; Scott et al. 2011). Similarly, Leggate et al. (2010) reported moderate and high intensity intermittent cycling stimulated a comparable increase in IL-6 immediately post exercise, which gradually returned to baseline at $23 \mathrm{~h}$. Normally, post-exercise cytokine kinetics are dictated by exercise intensity, with prolonged strenuous bouts stimulating a greater response, compared to shorter intense bouts (Suzuki 2018). Plasma IL-6, derived from skeletal muscle, usually increases exponentially immediately post exercise, stimulating an antiinflammatory cascade, giving rise to IL-1ra, IL-10 while blunting TNF- $\alpha$ (Petersen and Pedersen 2005). Thus, a rise in post-exercise IL-6 has evolved into an important modulating agent in immunosuppression, however, this is not a consistent finding in the current study, as TNF- $\alpha$ exhibits different kinetics, increasing post exercise in both trials and remaining elevated in the circulation for longer. This is not the first reports of such a rise, as Scott et al. (2011) reported TNF- $\alpha$ increased following 60 min of moderate and vigorous exercise. Numerous mechanisms may account for this rise in IL-6 including reduced glycogen availability, energy expenditure or changes in calcium and stress hormone secretion (i.e. catecholamines, growth factors or cortisol) (Peake et al. 2007). While the current findings are consistent with exercise-induced muscular IL-6 secretion and subsequent appearance in the vasculature, perhaps the unaccustomed fast treadmill walking simply stimulated a pro-inflammatory response characterised by the secretion of TNF- $\alpha$ (Ostrowski et al. 2001). It has also been hypothesised that blood sampling methods may also stimulate such a reaction (Haack et al. 2002). Dixon and colleagues (2009) compared sampling techniques and reported blood drawn via cannula caused a fourfold increase in pro-inflammatory mediators compared to marginal changes with venepuncture. Much of this response is understood to stem from a local inflammatory response within the indwelling vein and may account for the sustained elevation in TNF- $\alpha$ during the post-exercise period, whereas the exercise-induced IL-6 response gradually normalised (Thompson and Dixon 2009). Typically, TNF- $\alpha$ mediates systemic inflammation and is expressed in response to infection or tissue injury (Cawthorn and Sethi 2008). In many respects, the same cytokines and chemokines are released during the acute phase response but differ in the order of activation, starting with TNF- $\alpha$ (Petersen and Pedersen 2005). Within this pro-inflammatory model, neutrophil chemotaxis upregulates as does the expression of adhesion molecules, selectins and chemokines, exacerbating the pro-inflammatory state (Mihara et al. 2012). Perhaps the inclusion of a resting control group might have provided clarity on the source of cytokine secretion, but this aside, the current study is the first to show a bout of HIIW has such an effect and also highlights the complexities in analysing cytokine networking and inflammatory regulation post exercise.

ROS may also prove a key mediator within the exercise-induced cytokine response through the stimulation of $\mathrm{NF}-\mathrm{\kappa B}$, which subsequently regulates inflammation (Kramer and Goodyear 2007). Steinberg et al. (2007) provided data for a possible interaction after reporting an accompanied rise in lipid peroxidation and cytokine secretion. However, the current study showed no such changes in biomarkers of oxidative stress indicating the mechanism with walking may be more complex. It is possible that the exercise intensities increased cytokine concentrations independent of oxidative stress, or perhaps oxidative parameters outside the scope of this study may have contributed. Moreover, the activation of mitogen-activated protein kinases changes in calcium homeostasis, or impaired glucose availability may also attribute to the cytokine response (Fischer 2006). As participants undertook exercise while fasting the latter might be possible, coordinated via AMP-activated protein kinase signalling (Li and Gleeson 2005).

HIIW did not promote an increase in ROS or other related biomarkers. Several studies reported increased free radical production and oxidative damage with vigorous to maximal bouts of exercise (Alessio et al. 2000; Quindry et al. 2003; Davison et al. 2012). It has also been reported that as little as $5 \mathrm{~min}$ of exercise at $70 \% \dot{\mathrm{V}} \mathrm{O}_{2}$ max can stimulate oxidative damage (Fogarty et al. 2011). Thus, the exercise intensity, duration and mode appear to be key mediating factors for the production of oxidative stress (Parker et al. 2014). Whilst humans are equipped with a sophisticated network of antioxidant defence mechanisms designed to neutralise ROS, an intense or extended bout can lead to lipid, protein and/or DNA damage (Valko et al. 2007; Powers and Jackson 2008). Perhaps the exercise trials employed did not sufficiently overwhelm antioxidant capacity, preventing a rise in oxidative stress and protection against molecular damage. Alternatively, several studies have reported greater antioxidant status in trained participants (Powers and Jackson 2008; Djordjevic et al. 2012), indicating that current participants may possess efficient antioxidant capability, allowing them to manage ROS more effectively, given the enzymatic antioxidant response associated with exercise training. The observed reduction in lycopene following HIIW provides the best example of this antioxidant action. Lycopene has the capacity to neutralise potent-free radicals (hydroxyl, hypochlorous acid and peroxyl), offering protection against lipid peroxidation and lipoprotein modification while stimulating enzymatic antioxidants (Pennathur et al. 2010). 
Scavenging peroxyl radicals may preserve $\alpha$-tocopherol, a prominent chain-breaking antioxidant, possibly translating to the main effect for time at 2 and $4 \mathrm{~h}$ (Stahl and Sies 2003; Powers and Jackson 2008). Davison et al. (2012) demonstrated the importance of $\alpha$-tocopherol, as a reduction prompted a rise in lipid peroxidation and free radical production. Interestingly, IL-6 is known to upregulate enzymatic antioxidant expression, so it may conceivably mobilise nonenzymatic antioxidants, given its proposed role in adipose tissue and adipokine/myokine crosstalk (Vassilakopoulos et al. 2003; Trayhurn et al. 2011). Overall, the lack of change in oxidative parameters, some of which are potent signalling molecules (i.e. $\mathrm{H}_{2} \mathrm{O}_{2}$ ), could mean the acute activation of some redox-sensitive adaptations are missed (Veal et al. 2007). Perhaps the exercise-induced increase in cytokine activity was triggered by minimal, subtle changes in ROS, or by other redox variables, not directly evident in the current study. Further, the precise post-exercise sampling time points may account for the negligible change, as oxidative parameters are variable in nature and respond to stimuli differently over time (Michailidis et al. 2007). Alternatively, the results could potentially suggest another mechanism of action, at least in this exercise model, though at this time it is difficult to conclude and further research, possibly including analyses of muscle tissue, may be necessary to provide more mechanistic insight.

Finally, a main effect for time was detected for endothelin-1. Endothelin-1 participates in vasoconstriction, free radical production, proliferation and platelet activation (Böhm and Pernow 2007). Therefore, determining its acute function is desirable as previous research has mainly focused on aerobic exercise training (Maeda et al. 2009). Within the acute exercise model, McClean et al. (2015) also reported endothelin-1 increased immediately following $30 \mathrm{~min}$ of aerobic exercise at $55 \% \dot{V} \mathrm{O}_{2}$ max. The rationale for the increase may be associated with other vasoactive or pro-inflammatory mediators or exercise-induced changes in catecholamines but further research is necessary to explore this pathway. The findings suggest that the increase in endothelin- 1 and its associated detrimental effects are overridden as improvements in vascular function persist.

This randomised control trial is not without limitations. First, including a resting control group within the experimental design would add clarification to the inflammatory response, attributing changes to the sampling method or indeed the bouts of exercise. IL-6 is a responsive cytokine but our analysis and subsequent interpretation is based on its perceived post-exercise anti-inflammatory actions, as such this could be considered a minor limitation. Furthermore, analysing a more diverse array of cytokines (namely IL1ra and IL-10) during the post-exercise period would allow a comprehensive investigation of the systemic and delayed inflammatory response. Second, stringent dietary control and analyses might provide a commentary on glycogen availability as well as antioxidant status, given both interfere in signalling processes that regulate cytokine secretion. Likewise, sleep quality has recently emerged as potential influence over markers of immunity and as such should be considered carefully in the design of studies addressing cytokine activity and exercise. Future investigations should place an emphasis on clinical or perhaps elderly populations, given global improvements in life expectancy and the influence of inflammation during ageing. Finally, further trials should attempt to clarify the intracellular mechanisms relating to oxidative stress and cytokine activity within the exercise model.

In summary, the results indicate that walking modulates systemic cytokine secretion independent of oxidative stress. Walking also appears to promote an antioxidant response and collectively improves vascular function. Theoretically, the findings (i.e. no major changes in inflammatory or oxidative parameters) suggest HIIW and CMW are equally effective and stimulate similar physiological responses. Given time commitments are often cited for inactivity, HIIW may provide an alternative method for engaging in daily physical exertion. Further research is necessary to establish the precise mechanisms of action as well as determining the longitudinal efficacy of intermittent bouts of walking within the broader spectrum of public health.

Acknowledgements This research was completed as part of a Research Scholarship awarded by Ulster University.

Author contributions $\mathrm{MB}, \mathrm{CM}, \mathrm{GD}$ and MM conceived and designed the research. MB collected the data. $\mathrm{MB}$ and JB completed the analysis. $\mathrm{MB}, \mathrm{CM}$ and GD conducted the statistical analysis. MB prepared the manuscript. CM, GD and MM reviewed the manuscript. All authors read and approved the final version.

\section{Compliance with ethical standards}

Conflict of interest None of the authors have a professional relationship with companies or manufacturers that might benefit from the results of the present study.

Open Access This article is distributed under the terms of the Creative Commons Attribution 4.0 International License (http://creativeco mmons.org/licenses/by/4.0/), which permits unrestricted use, distribution, and reproduction in any medium, provided you give appropriate credit to the original author(s) and the source, provide a link to the Creative Commons license, and indicate if changes were made.

\section{References}

Alessio HM, Hagerman AE, Fulkerson BK, Ambrose J, Rice RE, Wiley RL (2000) Generation of reactive oxygen species after exhaustive aerobic and isometric exercise. Med Sci Sports Exerc $32: 1576-1581$ 
Altman DG (1980) Statistics and ethics in medical research: III How large a sample? Br Med J 281:1336-1338

Böhm F, Pernow J (2007) The importance of endothelin-1 for vascular dysfunction in cardiovascular disease. Cardiovasc Res 76:8-18

Boutcher SH (2011) High-intensity intermittent exercise and fat loss. J Obes. https://doi.org/10.1155/2011/868305

Cawthorn WP, Sethi JK (2008) TNF-alpha and adipocyte biology. FEBS Lett 582:117-131

Chu WM (2013) Tumor necrosis factor. Cancer Lett 328:222-225

Davison GW, Ashton T, McEneny J, Young IS, Davies B, Bailey DM (2012) Critical difference applied to exercise-induced oxidative stress: the dilemma of distinguishing biological from statistical change. J Physiol Biochem 68:377-384

Dixon NC, Hurst TL, Talbot DC, Tyrrell RM, Thompson D (2009) Active middle-aged men have lower fasting inflammatory markers but the postprandial inflammatory response is minimal and unaffected by physical activity status. J Appl Physiol 107:63-68

Djordjevic DZ, Cubrilo DG, Puzovic VS, Vuletic MS, Zivkovic VI, Barudzic NS, Radovanovic DS, Djuric DM, Jakovljevic VL (2012) Changes in athletes redox state induced by habitual and unaccustomed exercise. Oxid Med Cell Longev. https://doi. org/10.1155/2012/805850

Fischer CP (2006) Interleukin-6 in acute exercise and training: what is the biological relevance? Exerc Immunol Rev 12:6-33

Fogarty MC, Hughes CM, Burke G, Brown JC, Trinick TR, Duly E, Bailey DM, Davison GW (2011) Exercise-induced lipid peroxidation: implications for deoxyribonucleic acid damage and systemic free radical generation. Environ Mol Mutagen 52:35-42

Francois ME, Little JP (2015) Effectiveness and safety of highintensity interval training in patients with type 2 diabetes. Res Pract 28:39-44

Gibala MJ, Little JP, Macdonald MJ, Hawley JA (2012) Physiological adaptations to low-volume, high-intensity interval training in health and disease. J Physiol 590:1077-1084

Gleeson M, Bishop NC, Stensel DJ, Lindley MR, Mastana SS, Nimmo MA (2011) The anti-inflammatory effects of exercise: mechanisms and implications for the prevention and treatment of disease. Nat Rev Immunol 11:607-615

Haack M, Kraus T, Schuld A, Dalal M, Koethe D, Pollmacher T (2002) Diurnal variations of interleukin-6 plasma levels are confounded by blood drawing procedures. Psychoneuroendocr 27:921-931

Harris RA, Padilla J, Hanlon KP, Rink LD, Wallace JP (2008) The flow-mediated dilation response to acute exercise in overweight active and inactive men. Obes 16:578-584

He F, Li J, Liu Z, Chuang C-C, Yang W, Zuo L (2016) Redox mechanism of reactive oxygen species in exercise. Front Physiol. https ://doi.org/10.3389/fphys.2016.00486

Hood MS, Little JP, Tarnopolsky MA, Myslik F, Gibala MJ (2011) Low-volume interval training improves muscle oxidative capacity in sedentary adults. Med Sci Sports Exerc 43:1849-1856

Howley ET, Bassett DR, Welch HG (1995) Criteria for maximal oxygen uptake: review and commentary. Med Sci Sports Exerc 27:1292-1301

Kefaloyianni E, Gaitanaki C, Beis I (2006) ERK 1/2 and p38-MAPK signalling pathways, through MSK1, are involved in NF- $\mathrm{KB}$ transactivation during oxidative stress in skeletal myoblasts. Cell Signal 18:2238-2251

Kramer HF, Goodyear LJ (2007) Exercise, MAPK, and NF-кB signalling in skeletal muscle. J Appl Physiol 103:388-395

Leggate M, Nowell MA, Jones SA, Nimmo MA (2010) The response of interleukin- 6 and soluble interleukin- 6 receptor isoforms following intermittent high intensity and continuous moderate intensity cycling. Cell Stress Chaperones 15:827-833

Li TL, Gleeson M (2005) The effects of carbohydrate supplementation during the second of two prolonged cycling bouts on immunoendocrine responses. Eur J Appl Physiol 95:391-399
Little JP, Gillen JB, Percival ME, Safdar A, Tarnopolsky MA, Punthakee Z, Jung ME, Gibala MJ (2011a) Low-volume highintensity interval training reduces hyperglycemia and increases muscle mitochondrial capacity in patients with type 2 diabetes. J Appl Physiol 111:1554-1560

Little JP, Safdar A, Bishop D, Tarnopolsky MA, Gibala MJ (2011b) An acute bout of high-intensity interval training increases the nuclear abundance of PGC- $1 \alpha$ and activates mitochondrial biogenesis in human skeletal muscle. Am J Physiol Regul Integr Comp Physiol 300:1303-1310

Maeda S, Sugawara J, Yoshizawa M, Otsuki T, Shimojo N, Jesmin S, Ajisaka R, Miyauchi T, Tanaka H (2009) Involvement of endothelin-1 in habitual exercise-induced increase in arterial compliance. Acta Physiol 196:223-229

McClean C, Harris RA, Brown M, Brown JC, Davison GW (2015) Effects of exercise intensity on post-exercise endothelial function and oxidative stress. Oxid Med Cell Longev. https://doi. org/10.1155/2015/723679

Mendham AE, Donges CE, Liberts EA, Duffield R (2011) Effects of mode and intensity on the acute exercise-induced IL- 6 and CRP responses in a sedentary, overweight population. Eur J Appl Physiol 111:1035-1045

Michailidis Y, Jamurtas AZ, Nikolaidis MG, Fatouros IG, Koutedakis Y, Papassotiriou I, Kouretas D (2007) Sampling time is crucial for measurement of aerobic exercise-induced oxidative stress. Med Sci Sports Exerc 39:1107-1113

Mihara M, Hashizume M, Yoshida H, Suzuki M, Shiina M (2012) IL-6/IL-6 receptor system and its role in physiological and pathological conditions. Clin Sci 122:143-159

Murphy MH, Nevill AM, Murtagh EM, Holder RL (2007) The effect of walking on fitness, fatness and resting blood pressure: a metaanalysis of randomised, controlled trials. Prev Med 44:377-385

Nieman DC, Henson DA, Austin MD, Brown VA (2005) Immune response to a 30-minute walk. Med Sci Sports Exerc 37:57-62

Ost M, Coleman V, Kasch J, Klaus S (2016) Regulation of myokine expression: role of exercise and cellular stress. Free Radic Biol Med 98:78-89

Ostrowski K, Rohde T, Asp S, Schjerling P, Pedersen BK (2001) Chemokines are elevated in plasma after strenuous exercise in humans. Eur J Appl Physiol 84:244-245

Parker L, McGuckin TA, Leicht AS (2014) Influence of exercise intensity on systemic oxidative stress and antioxidant capacity. Clin Physiol Funct Imaging 34:377-383

Peake JM, Suzuki K, Coombes JS (2007) The influence of antioxidant supplementation on markers of inflammation and the relationship to oxidative stress after exercise. J Nutr Biochem $18: 357-371$

Peake JM, Della Gatta P, Suzuki K, Nieman DC (2015) Cytokine expression and secretion by skeletal muscle cells: regulatory mechanisms and exercise effects. Exerc Immunol Rev 21:8-25

Pennathur S, Maitra D, Byun J, Sliskovic I, Abdulhamid I, Saed GM, Diamond MP, Abu-Soud HM (2010) Potent antioxidative activity of lycopene: a potential role in scavenging hypochlorous acid. Free Radic Biol Med 49:205-213

Petersen AM, Pedersen BK (2005) The anti-inflammatory effect of exercise. J Appl Physiol 98:1154-1162

Powers SK, Jackson MJ (2008) Exercise-induced oxidative stress: cellular mechanisms and impact on muscle force production. Physiol Rev 88:1243-1276

Powers SK, Talbert EE, Adhihetty PJ (2011) Reactive oxygen and nitrogen species as intracellular signals in skeletal muscle. J Physiol 589:2129-2138

Quindry JC, Stone WL, King J, Broeder CE (2003) The effects of acute exercise on neutrophils and plasma oxidative stress. Med Sci Sports Exerc 35:1139-1145 
Radak Z, Zhao Z, Koltai E, Ohno H, Atalay M (2013) Oxygen consumption and usage during physical exercise: the balance between oxidative stress and ROS-dependent adaptive signalling. Antioxid Redox Signal 18:1208-1246

Sallam N, Laher I (2016) Exercise modulates oxidative stress and inflammation in aging and cardiovascular diseases. Oxid Med Cell Longev. https://doi.org/10.1155/2016/7239639

Scheele C, Nielsen S, Pedersen BK (2009) ROS and myokines promote muscle adaptation to exercise. Trends Endocr Metab 20:95-99

Scott JP, Sale C, Greeves JP, Casey A, Dutton J, Fraser WD (2011) Effect of exercise intensity on the cytokine response to an acute bout of running. Med Sci Sports Exerc 43:2297-2306

Sies H (2015) Oxidative stress: a concept in redox biology and medicine. Redox Biol 4:180-183

Stahl W, Sies H (2003) Antioxidant activity of carotenoids. Mol Asp Med 24:345-351

Steinberg JG, Ba A, Bregeon F, Delliaux S, Jammes Y (2007) Cytokine and oxidative responses to maximal cycling exercise in sedentary subjects. Med Sci Sports Exerc 39:964-968

Suzuki K (2018) Cytokine response to exercise and its modulation. Antioxid. https://doi.org/10.3390/antiox7010017

Thompson D, Dixon N (2009) Measurement of postprandial interleukin- 6 by using a catheter: what does it tell us? Am J Clin Nutr 90:1446-1447
Thurnham DI, Smith E, Flora PS (1988) Concurrent liquid-chromatographic assay of retinol, alpha-tocopherol, beta-carotene, alpha-carotene, lycopene, and beta-cryptoxanthin in plasma, with tocopherol acetate as internal standard. Clin Chem 34:377-381

Trayhurn P, Drevon CA, Eckel J (2011) Secreted proteins from adipose tissue and skeletal muscle-adipokines, myokines and adipose/ muscle cross-talk. Arch Physiol Biochem 117:47-56

Valko M, Leibfritz D, Moncol J, Cronin MT, Mazur M, Telser J (2007) Free radicals and antioxidants in normal physiological functions and human disease. Int J Biochem Cell Biol 39:44-84

Vassilakopoulos T, Karatza MH, Katsaounou P, Kollintza A, Zakynthinos S, Roussos C (2003) Antioxidants attenuate the plasma cytokine response to exercise in humans. J Appl Physiol 94:1025-1032

Veal EA, Day AM, Morgan BA (2007) Hydrogen peroxide sensing and signaling. Mol Cell 26:1-14

Welc SS, Clanton TL (2013) The regulation of interleukin-6 implicates skeletal muscle as an integrative stress sensor and endocrine organ. Exp Physiol 98:359-371 